\title{
Interorganizational Knowledge Exchanges
}

\author{
Dimitris Apostolou, Gregoris Mentzas, Bertin Klein, Andreas Abecker, \\ and Wolfgang Maass, INKASS
}

\section{The proposed}

\section{knowledge exchange}

system couples case-

based reasoning with

ontologies to assist

match-making between

knowledge offers and

knowledge demands in

an interorganizational

context.

1 he desire for knowledge exchange between business entities has various causes. Consulting companies, for example, want to make money by selling their expertise. Other possible motives include wanting to deliver a more sophisticated service to existing customers, attract new customers by expanding the market reach, or generate

additional revenues by selling knowledge on top of complex tangible products.

Knowledge exchange systems (KESs) are an emerging niche in the knowledge management mar$\operatorname{ket}^{1}$ that let organizations offer their knowledge as a product to internal and external customers. KESs manage the workflow of posed questions, route the questions to experts, and can enhance transactional sites by providing knowledgeable responses and content to inquiries.

Existing KESs, which typically employ keywordbased search engines, retrieve content that often is irrelevant, is out of context, or has no proper granularity. ${ }^{2}$ A knowledge asset offered on a KES isn't like a book that can be described and retrieved with simple keyword retrieval. It has manifold complex context- and content-related features that determine its applicability and usefulness in a given situation. Moreover, the administration of content in existing KESs is limited to the typical functionalities of portal or content management systems, such as identifying broken links and document versioning.

KESs need advanced tools that analyze users' diverse interactions, help administrators improve the organization of content, and provide hints for producing new content or improving existing content.
Furthermore, a key lesson learned from the discipline of knowledge management is that knowledge has both an explicit dimension and a harder-toarticulate tacit dimension. So, a KES should provide not only access to explicit knowledge but also links to human experts.

INKASS (Intelligent Knowledge Assets Sharing and Trading) is a KES for implementing interorganizational knowledge exchanges. In designing InKASS, we concentrated on representing and retrieving knowledge assets in the user's context. To satisfy this requirement, INKASs employs a generic ontology-based approach for representing and exchanging knowledge assets. In addition, it includes a suite of tools that maintain and improve knowledge exchange by taking into account users' interactions with the system. Empirical evaluations of the system's perceived usefulness show that INKASS provides a useful platform for interorganizational knowledge exchanges with effective content improvement.

\section{Ontologies and typical}

\section{knowledge exchange practices}

Knowledge exchanges broadly include practices similar to those involved in shopping. The shopping 


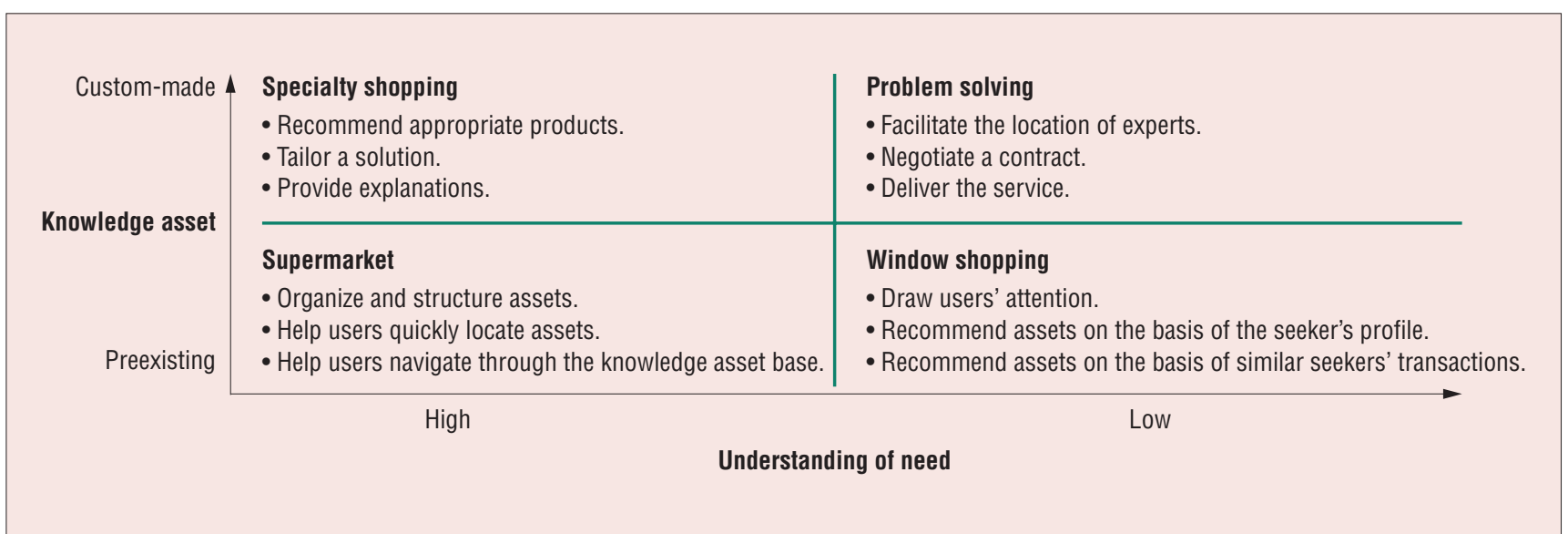

Figure 1. The four shopping styles. These styles form the basis for an understanding of knowledge exchange practices.

metaphor helps us relate knowledge exchanges to our experiences with typical shopping practices (for example, displays or shop windows for advertising and demonstrating products). Shoppers often have a specific demand but often don't know beforehand which product will meet it.

This situation is particularly true for knowledge seekers. Often they need to solve a problem but can't say, or don't even know, what knowledge asset can help them solve it. Even worse, just as with many other products, some knowledge assets might be crafted only after the customer has ordered them and paid for them. So, these knowledge assets must be offered with a descriptive package and label. Moreover, the offer needs to induce the buyer to trust that he or she is about to buy a high-quality, and thus valuable, product.

By looking at everyday shopping habits, we can distinguish four shopping styles according to the knowledge seeker's understanding of his or her need and the preexistence of knowledge assets (see Figure 1).

We call the first style supermarket. People visit supermarkets to buy their everyday items. They know what they need and where to get it (for example, they quickly move to the dairy product shelves and select their favorite brand of cheese).

On the knowledge exchange, a shelf can be a class in an ontology. (For a description of the INKASS information ontology, see the "Knowledge Asset Representation and Similarity-Based Matching" sidebar.) The ontology's classes are the labels assigned to knowledge assets for classification. Furthermore, the knowledge exchange can visualize the ontology to help knowledge seekers navigate through large numbers of knowledge assets.

The second style is specialty shopping. In this case, people visit a specialty shop that has the expertise and capabilities to provide customized solutions. A specialist or craftsman helps them elaborate their understanding of the problem and recommends appropriate products or tailors a solution to their needs. Furthermore, the specialist provides ample clarifications and answers questions to create trust.

The knowledge exchange can map the seeker's initial problem description to terms of the ontology and report to the seeker, presenting results matching the problem description. The knowledge exchange can further use the ontology to interactively detail the problem description by calculating information gains until the seeker finds the right knowledge asset. During this process, the knowledge exchange can explain the relation between the iterated query and the offered assets. So, the knowledge seeker can build trust by understanding alternative offers without having to access their content. In some cases, existing knowledge assets might not satisfy the seeker's needs. In such cases, the knowledge exchange can again use the ontology to model the descriptions of knowledge assets that might be produced to address the seeker's request.

The third style is window shopping. People stroll around a shopping mall, a product in a shop window attracts their attention, and they buy it. The customer's attentions can be attracted by a product's features, such as its appearance or applicability. The product might be something the customer didn't know existed, something a friend recommended, something the customer often thought about buying but never bought, or simply something advertised in the customer's local newspaper.

On a knowledge exchange, the prerequisites for window shopping can be achieved by tools that help optimize the ontological description of knowledge assets for knowledge seekers' mindsets. If knowledge seekers are willing to provide information about their business and domain background, they benefit in terms of service quality. In these cases, the knowledge exchange can direct knowledge offers to potential seekers on the basis of their profiles or contexts - that is, their business, their role in their organization, and their professional focus.

The final style is problem solving. In this situation, the customer locates an expert in the telephone directory and employs that expert to tailor a solution. The knowledge exchange can model experts as class instances in the ontology. So, they can be found "on the shelves" (that is, classes in the ontology). Employing an expert involves negotiation, contracting, and service delivery. The knowledge exchange can support these processes with workflows, ready to be executed by it.

A successful knowledge exchange should be able to serve all four shopping styles, especially in the current early state of knowledge exchanges. Today, for example, there are few standardized knowledge products that can be bought according to the supermarket style, comparable to buying something such as bread or milk. For any knowledge asset offer, knowledge seekers will probably use any of the four styles.

For example, think of an asset such as a one-day introductory course for telephone interviewer trainees. A large company might 
In the INKAss (Intelligent Knowledge Assets Sharing and Trading) knowledge exchange system (KES), each available knowledge asset comprises a core information object and a set of metadata describing that object. This description forms a case. Metadata are defined in the information ontology. ${ }^{1}$ The ontology comprises concepts and relationships between them that aim to describe not only a knowledge asset's content but also

- the context in which that asset can be used,

- the community of users it targets,

- its history record,

- possible evaluation aspects,

- business aspects such as the applicable pricing model, and

- intellectual-property-rights and security aspects.

An information object can appear in more than one case at the same time, similarly to a book that can be on both the science fiction and bestseller shelves. Some of the metadata will just have simple text as values, such as the title (attribute-value representation), whereas others might refer to other objects (structured representation). For example, the owner metadata might point to another object representing that person; that object could have a set of metadata such as that person's competencies and qualifications.

INKASS automatically translates a user's query into a query case, even if that query is formulated as free text. The system then calculates the query case's similarity to the stored cases. The most similar stored case is the most relevant to the user's question.

The key to a case-based approach's success is its ability to accurately estimate the similarity between metadata of different cases. InKAss derives similarity measures from the semantic approximation of ontological concepts. Semantic approximation utilizes the information ontology's hierarchical structure to evaluate concepts' semantic similarity. This similarity is based on subsumption relationships and on the distances between nodes in the information ontology.
For example, in Figure A, the user has submitted a query for radiation welding. INKASS considers any knowledge asset annotated with a concept that's a descendent of Radiation welding in the ontology (for example, Laser_welding and lon_beam welding) to be an exact match. This is logical because all descendants of Radiation_welding are specific types of radiation welding. Moreover, concept similarity is based on concept proximity: the closer two concepts are in the ontology, the more similar they are. For instance, Laser_welding is more similar to lon_beam_welding than to Electroslag_weilding because in the former case, there are fewer in-between nodes.

In realistic situations, automated calculation of similarities isn't sufficient. Consider, for example, a case in which a user doesn't know exactly what kind of radiation welding he or she is applying but does know it's radiation welding. A case about Image_welding would automatically match exactly the query described previously but not necessarily the user's needs if the user is actually applying electron-beam welding (Eb_welding in Figure A). Moreover, some concepts might be distant in the ontology but share characteristics that make them relevant in a particular knowledge-seeking scenario. So, there should be a way to directly enter similarity measures between arbitrary ontology concepts. Our approach defines cases and similarity measures on top of RDF and RDF Schema as classes and properties, respectively. (For a detailed description of the INKAss information ontology and implementation details, see www.imu.iccs.gr/projects/ inkass/information_ontology.pdf.)

\section{Reference}

1. A. Abecker, "Toward a Technology for Organizational Memories," IEEE Intelligent Systems, vol. 13, no. 3, 1998, pp. 40-48.

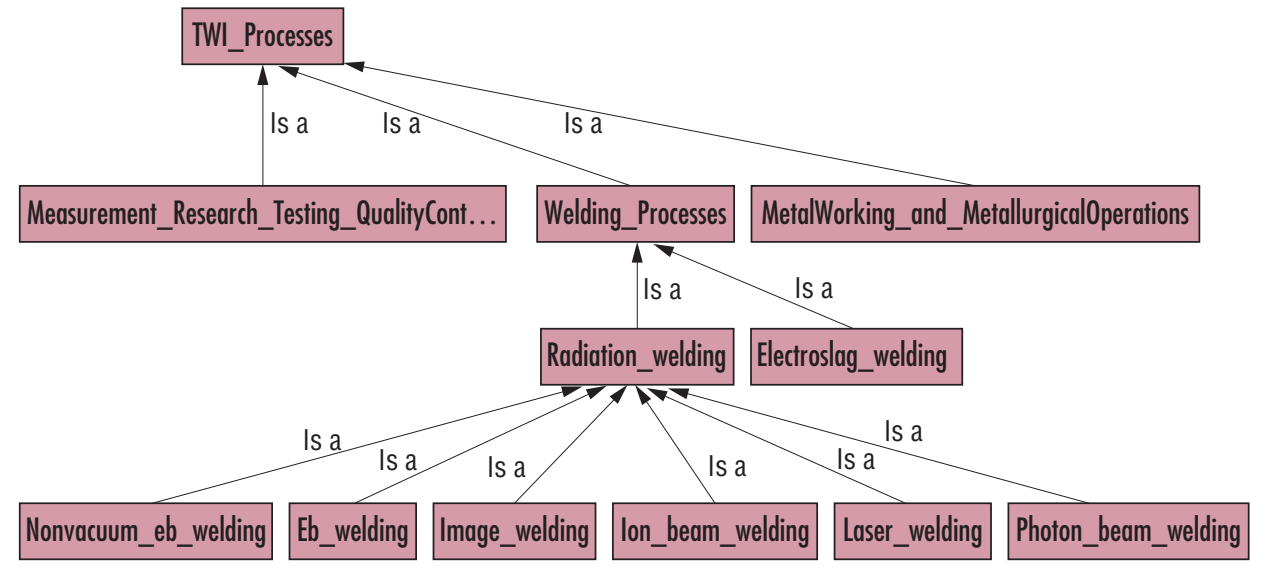

Figure A. A snapshot of a welding information ontology. Taxonomical relations can be used to evaluate semantic similarity of concepts. 


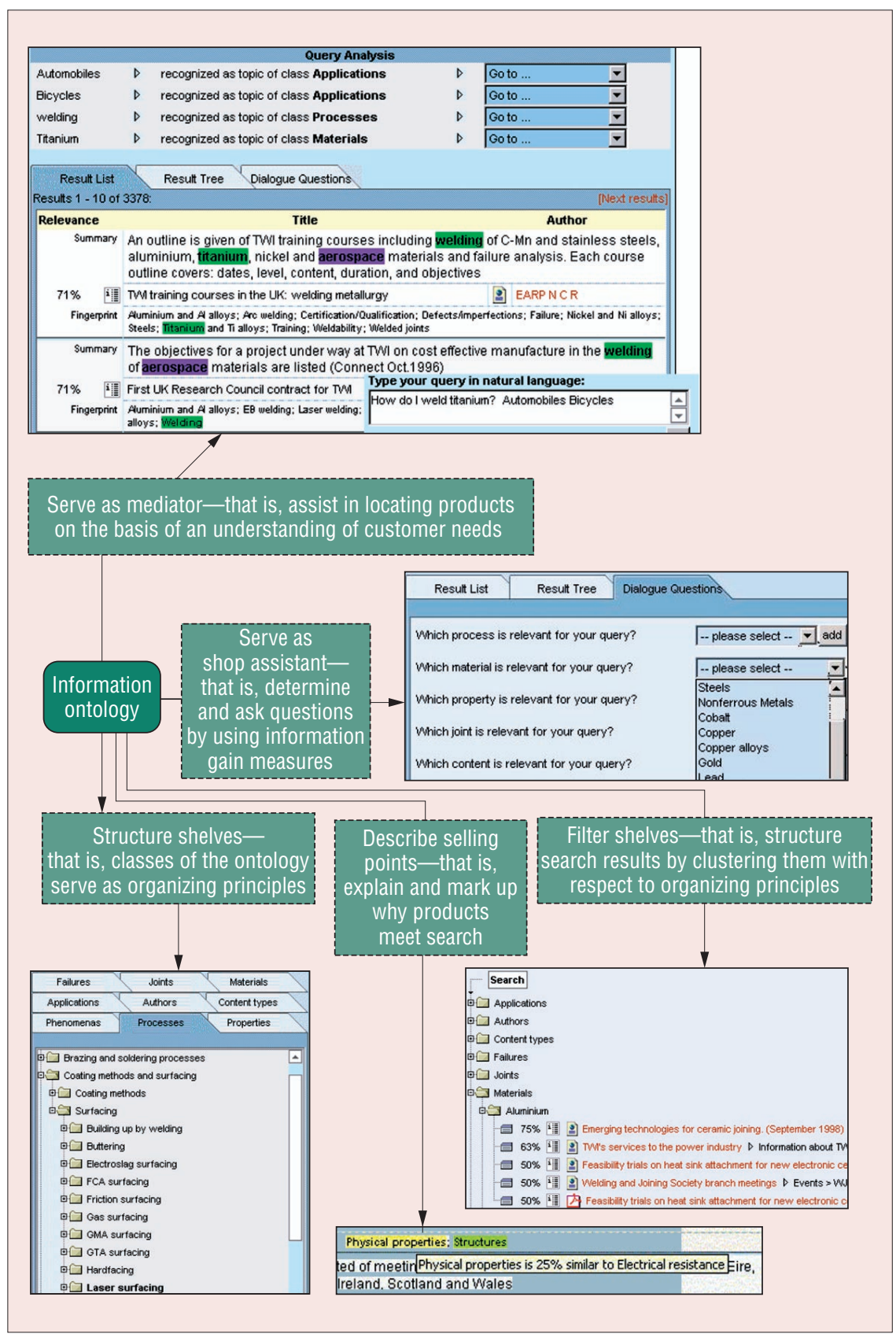

Figure 2. How the InKAss (Intelligent Knowledge Assets Sharing and Trading) information ontology helps users locate relevant knowledge offers. Knowledge exchanges require human-computer interaction techniques that help users express their needs, browse and query available offers, and elaborate their needs by interacting with the system.

book this course regularly, whenever they hire new staff (the supermarket style). Another company might make the strategic decision to invest in its employees' knowledge and actively search for the right offer (specialty shopping). Following a colleague's recommendation, the manager of a group of telephone interviewers might spontane- ously book the course (window shopping). A company selling such courses will surely be happy to attract all these styles of buyers. If a fourth kind of seeker discovers the company's expertise and asks for a slight adaptation of the course (problem solving), the company will likely be able to serve him or her.

\section{How Inkass supports knowledge exchanges}

Figure 2 shows how InKass uses its information ontology to offer five features that help knowledge seekers locate relevant knowledge offers.

The first feature is the mediator, which analyzes natural language queries. The system returns a list of matching offers and a list of keywords from the query that it understands (that is, keywords that are in the information ontology). These keywords provide feedback to the user on how the query relates to the ontology.

InKASS displays the offers as a configurable set of representative information ontology classes or properties-for example, author or descriptive summary. For single offers the customer can get more detailed previewing information and can trigger workflow processes (for example, ask for further assistance, ask for an offer, or buy). All actions are knowledge asset specific. For example, if the knowledge asset can be customized, the user can invoke a request-forproposal workflow to receive a quote specifically for his or her needs. If the knowledge asset is free, the user can directly download all related content.

The second feature is the shop assistant, which provides search improvement feedback. At any time the system provides context-sensitive dialogue questions. For example, it might ask which material is relevant to the query. When the user answers the question by selecting, for example, "steel," the system eliminates irrelevant retrieved offers and directs the user to more precise offers. The system rules out an offer only if the offer conflicts with the user's answer. Users can refine queries by selecting a value from a pull-down menu for each question. The system displays only questions that, when answered, identify the user context.

The third feature is shelf filtering, which navigates search results. At any time the user can switch to an alternative organization of the results. The list of offers can be structured on the basis of differentiating classes from the information ontology. For example, knowledge assets related to "project management" can be presented in categories such as "task scheduling," "resource usage," and "project planning." The system uses the information ontology to calculate and provide feedback about the available offers' dimensions. This is comparable 
to the feedback a casual customer receives when entering a shop, which typically has products displayed according to various categories.

The fourth feature is describing selling points, which explains search results. In the displayed text excerpts of the retrieved results, the system highlights matching keywords or synonyms (for example, synonyms such as "magnetite" when the user queried the concept "Fe3O4"). In approximate matches, the system returns results that either contain keywords or have been annotated with terms that are similar to the query keywords. To explain why these results are relevant, the system activates pop-up menus when the user moves the mouse over such a term. (The "Knowledge Asset Representation and Similarity-Based Matching" sidebar explains the relevance estimation mechanism.) Furthermore, the system presents the recognized keywords and their position in the ontology and provides the ability to retrieve knowledge assets clustered in the corresponding area of the ontology.

The final feature is shelf structuring. At any time the user can choose a navigation view, to navigate through the information ontology and browse for relevant content. The Topic Map menu offers two submenus. The Table View submenu presents all available topic categories in the form of tabs; each tab shows a category table with all subcategories. When the user clicks on a subcategory-for example, aluminumthe system displays a screen on the right where the user can view and edit the concepts and look for linked knowledge assets. Users can also subscribe to a notification service. The Map View submenu is an interactive, graphical visualization of parts of the information ontology. Here, the user can navigate and explore classes and knowledge assets linked to them, by clicking on the visible labels.

\section{Inkass overview}

To present the InKass functionalities, we utilize the Business Media Framework, ${ }^{3}$ which separates a business transaction into four phases.

In the information phase, a knowledge seeker expresses his or her knowledge needs. In INKASS, users can express their needs by means of

- standard full-text search, including phrase matching, arbitrary wild cards, and prox-

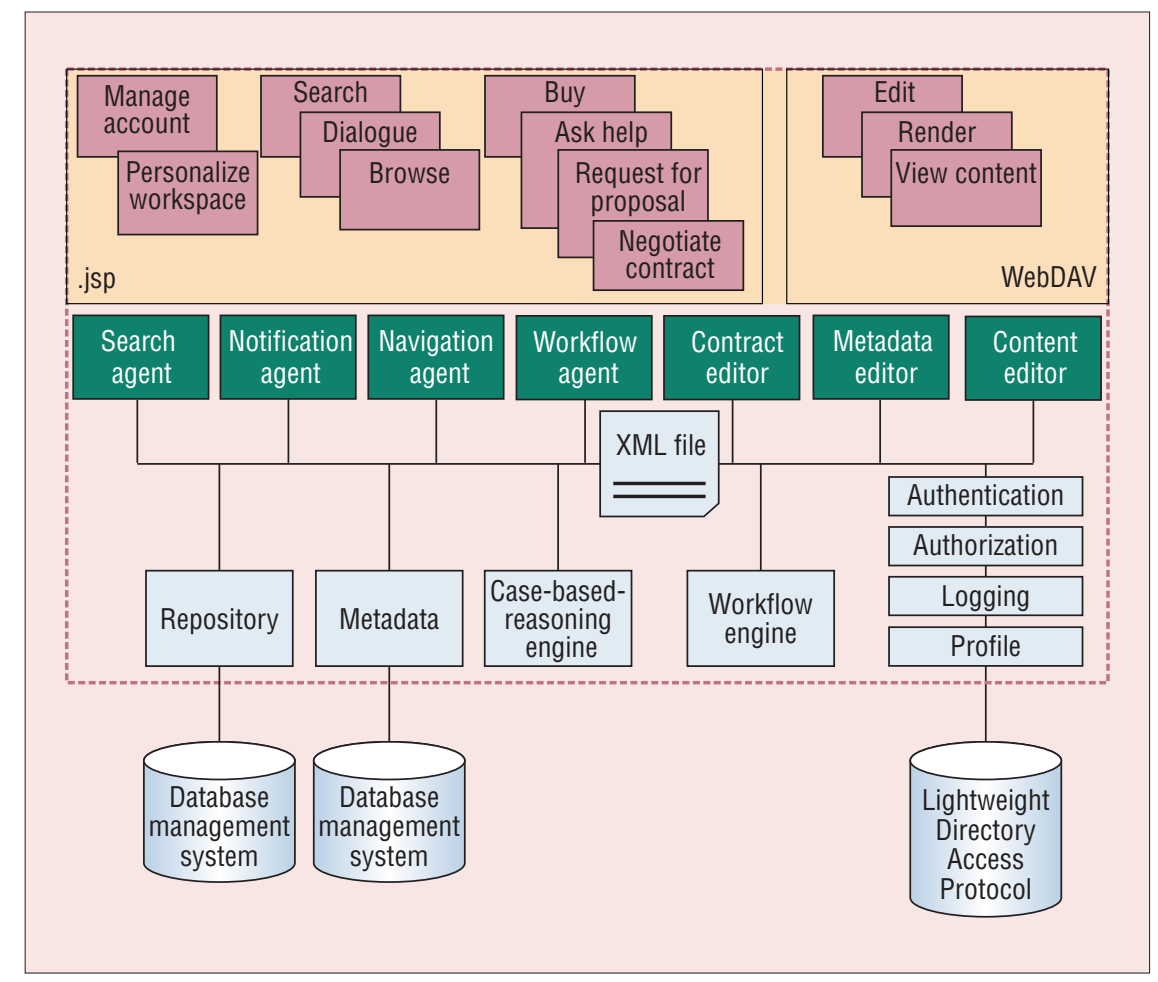

Figure 3. The InKAss system's three tiers. The top tier's components (orange) offer the required access services. The middle tier's core services (light blue) implement low-level functionalities such as security and metadata management. Higher-level agents (dark blue) combine the functionalities provided by the core services. For example, they handle the processes (such as storage versioning and search index update) involved with checking-in a new knowledge asset. The back-end tier (gray components) is the persistence layer.

imity search (for example, specifying that two keywords must appear next to each other);

- natural language queries in which the system utilizes language-processing methods (for example, stemming); and

- ontology-based similarity matching to resolve vague terms in queries.

In the intention phase, a knowledge seeker signals his or her intentions on the basis of the understanding gained in the information phase. InKASs employs a workflow engine that can implement several interaction modes (and corresponding pricing schemata) to suit the specific knowledge exchange needs. For example, it can implement a request-for-proposal workflow to help a knowledge seeker specify requirements and get a quote from the knowledge supplier.

The contracting phase applies to financially based knowledge exchanges and to cases involving creation of new knowledge. In this phase, knowledge seekers and suppliers negotiate contracts starting from the initial agreement reached in the intention phase. InKass provides a contract editor that supports definition of the contract terms applicable to a specific knowledge asset. This phase results in a legally binding contract that documents the knowledge supplier and buyer's mutually agreed obligations.

In the settlement phase, knowledge seekers and suppliers act according to the contract. This includes payment for and delivery of the knowledge asset. This phase results in the appropriate termination of the business transaction (that is, a successfully executed contract or a mutually agreed cancellation of the transaction). INKASS provides a workspace, shared between the supplier and the seeker, for submitting deliverables and communicating messages related to the work specified in the contract.

INKASS is built on top of the empolis three-tier content management suite (see Figure 3). 
Knowledge exchanges pose challenges to knowledge representation techniques, human-computer interaction approaches, and deployment methodologies.

\section{Ontology-based representation of cases}

Ontology-based content representation is becoming increasingly important for information retrieval in digital libraries, for Internet information search, and, more recently, for e-commerce. ${ }^{1}$ OWL is a semantic markup language for publishing and sharing ontologies on the Web. It's a vocabulary extension of RDF Schema that aims to overcome RDF Schema's limited expressive power.

Retrieving relevant ontology-represented content relies on exact, logic-based reasoning. However, a matchmaking mechanism on a knowledge exchange must be able to support the vague, uncertain matching processes between demand and requests, something a strict logic-based approach can't do. Case-based reasoning focuses on inexact reasoning by measuring the similarity between content objects. A detailed description of similarity-based matchmaking algorithms appears elsewhere. ${ }^{2}$ There are three main approaches for representing cases: ${ }^{2}$

- textual (representing cases in free text),

- conversational (representing cases as a list of questions and answers), and

- structural (representing cases according to a common domain model).

Our approach combines Semantic Web languages with case-based reasoning to represent and retrieve knowledge assets. It essentially exploits RDF and RDF Schema's ability to model heterogeneous, distributed Web resources and case-based reasoning's similarity-based retrieval.

\section{Human-computer interaction \\ and user feedback}

Expressing knowledge in a highly sophisticated domain (for example, technology consulting) with a multitude of available knowledge assets can be extremely difficult. This is particularly true when the user doesn't frequently work with the system. This is even truer because we have no clear information retrieval problem. The user's concrete demand often will be clarified as the user browses through the available content, or the demand might change owing to the offers provided. Electronic knowledge exchanges require humancomputer interaction techniques that help users express their needs, browse and query available offers, and adapt their needs to specific content features.

Gerard Salton and Michael McGill introduced relevance feedback to improve information retrieval precision. ${ }^{3}$ Relevance feedback employs two basic techniques: query expansion (adding new query terms) and term reweighting (modifying of query terms or weights). ${ }^{4}$ For example, LingoLogic is an interface technology that uses menus to progressively specify natural language queries. ${ }^{5}$ The user composes a sentence by either typing it in or selecting items from a cascade of menus driven by a grammar and predictive parser.

Relevance feedback has been used mainly to improve systems for image analysis and retrieval and textual information retrieval. For dealing with complex products and services, one of the most advanced approaches is IBM's Soft Navigation. ${ }^{6}$ In this approach, users explore and adapt their interests and preferences, adjust weights of specific attributes influenced by retrieval results, relax constraints, and add features required to locate the most appropriate insurance products.

Closer to our research is a goal-oriented method developed for incrementally improving organizational memories. ${ }^{7}$

\section{Improving knowledge exchange through user feedback}

Effectively producing one snapshot-like knowledge exchange isn't sufficient in reallife applications. A knowledge exchange should keep pace with users' rapidly changing knowledge needs and with possibly changing applications of existing knowledge assets. In general, improvement can be reactive or proactive. Reactive improvement occurs when the system generates a trigger and the administrator examines the trigger's cause. Proactive improvement occurs when the administrator investigates a possible root cause in specific content and fixes the problem.

The tools we developed support analysis of triggers and root causes to help the administrator make improvements (see Figure
4 on page 72). First, consider a user interaction with the KES that led to a successful transaction (a document download). The user downloaded the document "Develop Feasibility Study for Manufacturing Plants," which the system knew at that point to be useful only for designing a new manufacturing plant. By asking for aspects of the user's context that the user hasn't yet specified in the query, the system learned that this document is also useful for moving a plant. Essentially, this is new knowledge about knowledge-seeking problems and situations, solvable with available knowledge assets. The system translates this new knowledge into metadata, which in turn generate new cases for available information objects.

Now consider a failed user query (see Figure 5 on page 72). The administrator first uses the UserLog View, which helps determine why the query failed. This view shows which query terms have been identified and which information objects have been retrieved. If the system doesn't recognize some terms (they aren't in the ontology), it checks whether they're relevant and should become keywords. If they aren't relevant, the administrator adds them to the stopwords list using the Stopwords View. If some terms are relevant, the administrator checks whether they're in the ontology. If they are, the administrator simply annotates relevant information objects with these terms. If they aren't, the administrator enters them into the ontology using the Ontology View, manually establishes similarities between them and existing ontology concepts (if necessary), and accordingly annotates the relevant information objects.

To annotate information objects with 
The method consists of a general usage model, a set of indicators for improvement potential, and a cause-effect model. Our approach goes one step further in that it includes software tools to support specific improvement tasks.

\section{Instilling a market perspective into knowledge management}

Knowledge management isn't a single discipline; it's an integration of many fields. So, it's only natural that the scientific and popular literature presents many diverse methodologies for helping leverage an organization's knowledge assets. Such methodologies range from those facilitating the development of KM strategies ${ }^{8,9}$ to those assisting the life cycle of KM practice from awareness creation, to operational issues, to performance evaluation. ${ }^{10,11}$ Certain methods also tackle specific problems such as enabling knowledge creation and sharing within communities of practice, ${ }^{12}$ whereas others concentrate on KM's knowledge-engineering aspects. ${ }^{13,14}$

Our focus on interorganizational sharing and trading of knowledge led us to develop a methodology that adopts a different stance on KM. We concentrate on knowledge seekers and knowledge suppliers and also analyze characteristics of optional roles supporting a knowledge exchange (for example, knowledge brokers, archivists, escrow agents, knowledge publishers, and knowledge reviewers). We separate the knowledge exchange process into three phases: setup, implementation, and maintenance. This approach addresses the knowledge exchange's configuration and business model (that is, the value creation elements, the economic-control aspects, and the revenue sources). It also addresses how to guarantee that the knowledge has the proper usability and that the knowledge exchange matches the right knowledge assets between the knowledge provider and knowledge seeker.

\section{References}

1. D. Fensel, Ontologies: Silver Bullet for Knowledge Management and Electronic Commerce, Springer, 2001.

2. R. Bergmann, Experience Management: Foundations, Development Methodology, and Internet-Based Applications, LNAI 2432, Springer, 2002.

3. G. Salton and M.J. McGill, Introduction to Modern Information Retrieval, McGraw-Hill, 1983.

4. Y. Ishikawa, R. Subramanya, and C. Faloutsos, "Mindreader: Query Databases through Multiple Examples," Proc. 24th Int'l Conf. Very Large Databases (VLDB 98), Morgan Kaufmann, 1998, pp. 218-227; www.informedia.cs.cmu.edu/documents/ mindreader.pdf.

5. C.W. Thompson, P. Pazandak, and H.R. Tennant, "Talk to Your Semantic Web," IEEE Internet Computing, vol. 9, no. 6, 2005, pp. 75-79.

6. M. Stolze, "Soft Navigation in Electronic Product Catalogs," Int'l J. Digital Libraries, vol. 3, no. 1, 2000, pp. 60-66.

7. K.-D. Althoff, M. Nick, and C. Tautz, Improving Organizational Memories through User Feedback, tech. report 004.99/E, Frauenhofer Inst. Experimentelles Software Eng., 1999.

8. M. Earl, "Knowledge Management Strategies: Toward a Taxonomy," J. Management Information Systems, vol. 18, no. 1, 2001, pp. 215-233.

9. M. Zack, "Developing a Knowledge Strategy," California Management Rev., vol. 41, no. 3, 1999, pp. 125-145.

10. A. Tiwana, The Knowledge Management Toolkit: Practical Techniques for Building a Knowledge Management System, Prentice Hall, 2000.

11. G. Mentzas et al., Knowledge Asset Management: Beyond the Process-Centred and Product-Centred Approaches, Springer, 2002.

12. E. Wenger, R. McDermott, and W. Snyder, Cultivating Communities of Practice: A Guide to Managing Knowledge, Harvard Business School Press, 2002.

13. S. Staab et al., "Knowledge Processes and Ontologies," IEEE Intelligent Systems, vol. 16, no. 1, 2001, pp. 26-34.

14. G. Schreiber et al., Knowledge Engineering and Management: The CommonKADS Methodology, MIT Press, 1999. keywords, the administrator uses the Case Base View. This view lets the administrator sort information objects according to their statistical relevance to one or more keywords. For each information object, the system provides a measure of relevance to one or more keywords, utilizing a vector space algorithm. ${ }^{4}$ The administrator determines the relevance threshold for annotating the information object with the related keyword. Another helpful feature is sorting by information object similarity, which lets the administrator look for information objects that are statistically similar to a given information object.

\section{Evaluation}

We evaluated InKASS in three field trials with the Welding Institute (an international research and technology organiza- tion), Planet SA (a European management and technology consultancy), and the Athens Chamber of Commerce and Industry (not discussed here). To design and set up the knowledge exchanges, we applied the INKASS business-engineering methodology (see the "Related Work on Knowledge Exchanges" sidebar).

The field trials covered two evaluation phases over six months. They involved 25 clients of Planet and 100 members of the Welding Institute, representing different ages, IT literacy, and seniority. We aimed to assess the knowledge exchange's perceived usefulness and the improvement tools' effectiveness. Users could freely interact with the system but also had to perform a given set of test scripts. We assigned the scripts to force the limited number of users to access a specific subset of the offered knowledge assets, thereby providing enough user interaction data to test the tools.

We collected user feedback through a questionnaire and interviewed the users to gain deeper insight into their concerns about InKASS.

In the trial at the Welding Institute, we assessed the usefulness of INKASS versus the database system in operation during the trials. We found that 86 percent of users perceived that INKASS provided more relevant search results than the database system, 75 percent perceived that it had more useful functions, and 70 percent perceived that it was easier to use and faster.

In the trial at Planet, we assessed how improvements in the ontology, case base, and content base affected perception of the system's usefulness. To do so, we controlled other factors affecting perceived 


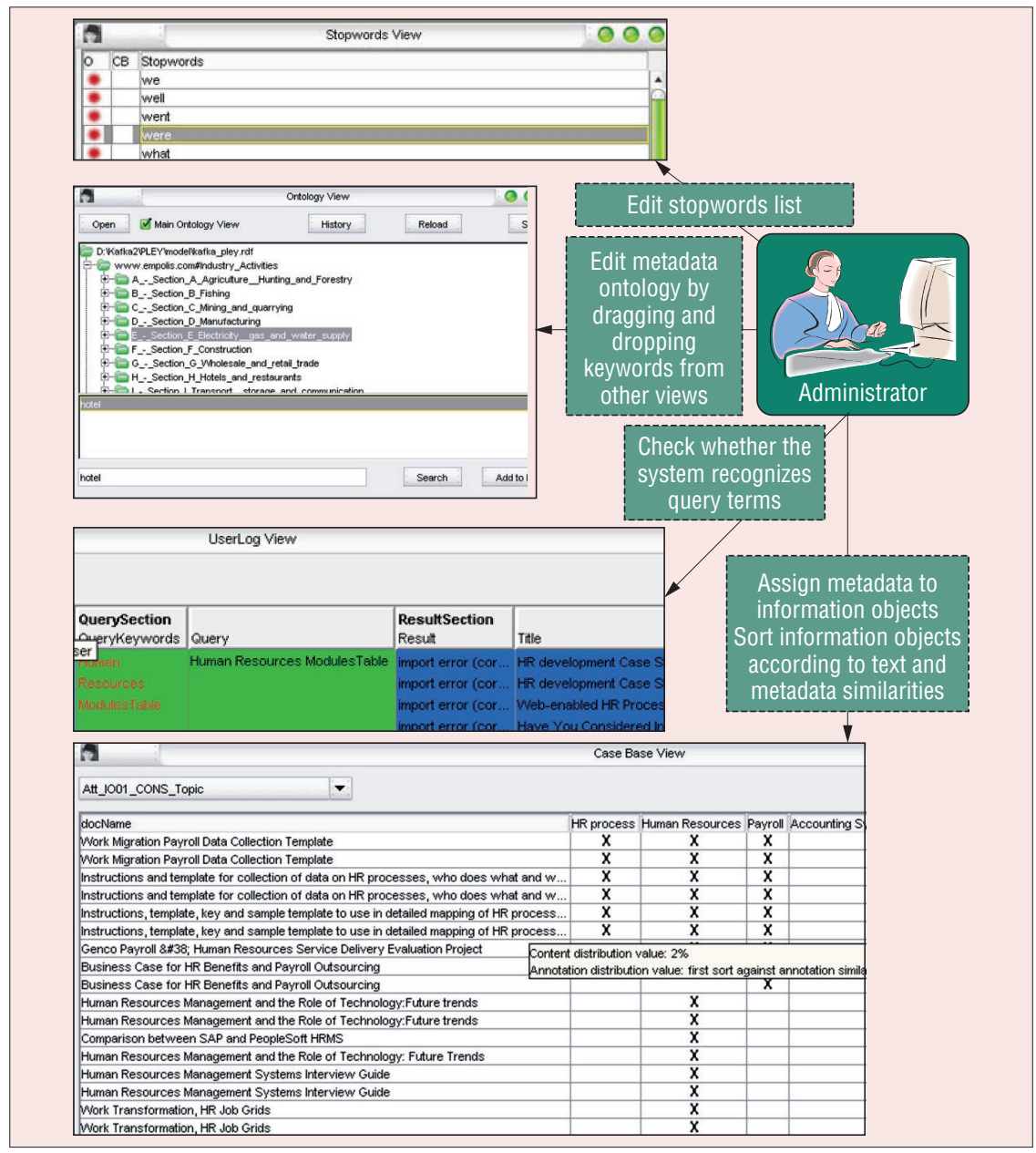

Figure 4 Knowledge exchange

improvement tools. These tools let system administrators analyze causes of problems and triggers for potential improvements.

usefulness such as the underlying content base's coverage. The results (see Figure 6) revealed that users perceived the improvements primarily when using the natural language query.

Figure 7 shows how, through user feedback, the tools improved the ontology, case base, and content base. There's also indication that the improvement rate and thus administrator effort decreased as the number of transactions increased.

$\mathbf{T}$ he development of INKASs has brought about four main innovations. First, it integrates intelligent search, retrieval, and assistance software with e-commerce functionalities, such as those needed to handle transactions and implement workflows (for example, a request for proposal).

Second, on the technical side, INKASs em-

Figure 5. Knowledge exchange improvement tasks. ${ }^{5}$ Triggers enable the administrator to perform actions that improve the knowledge exchange.

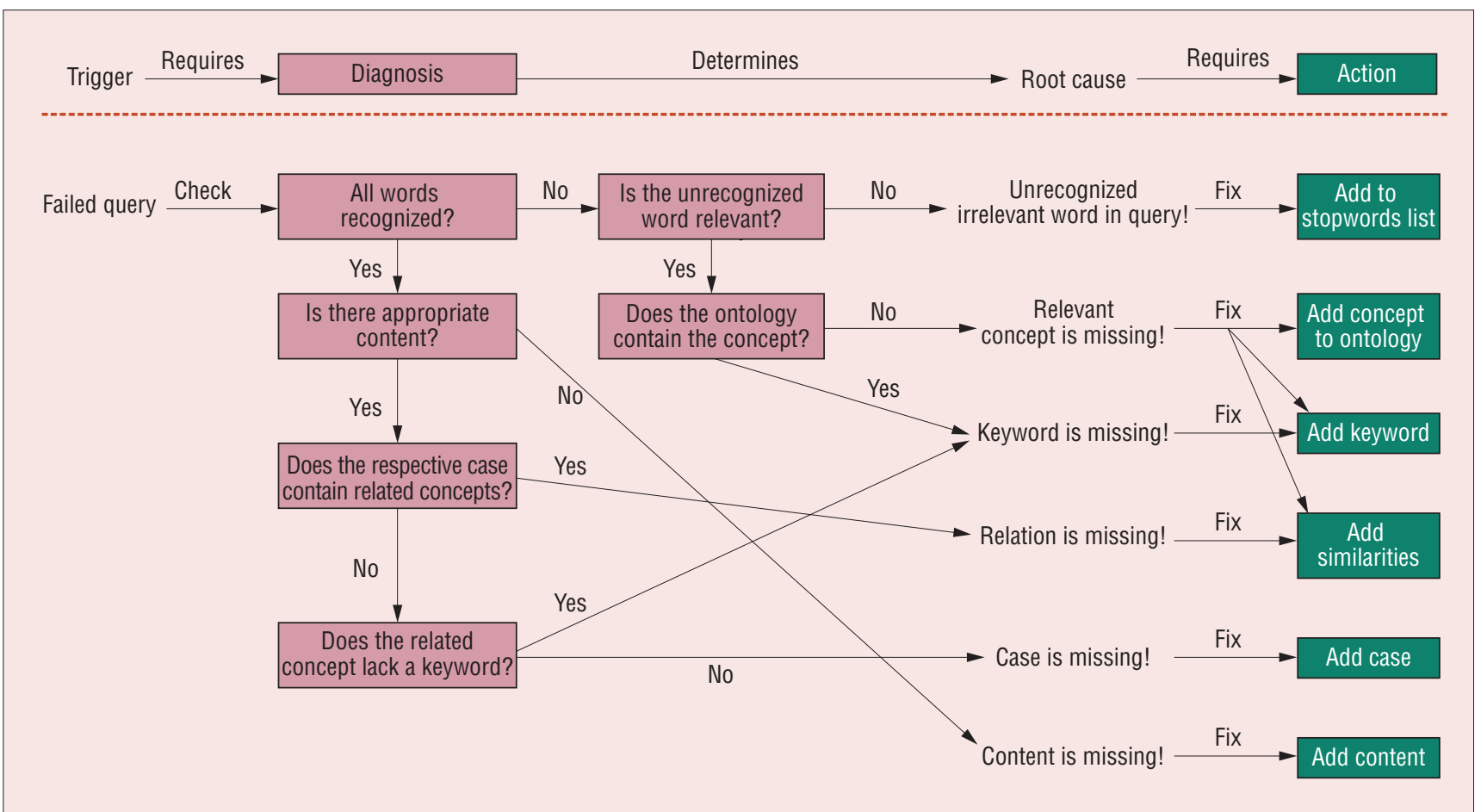




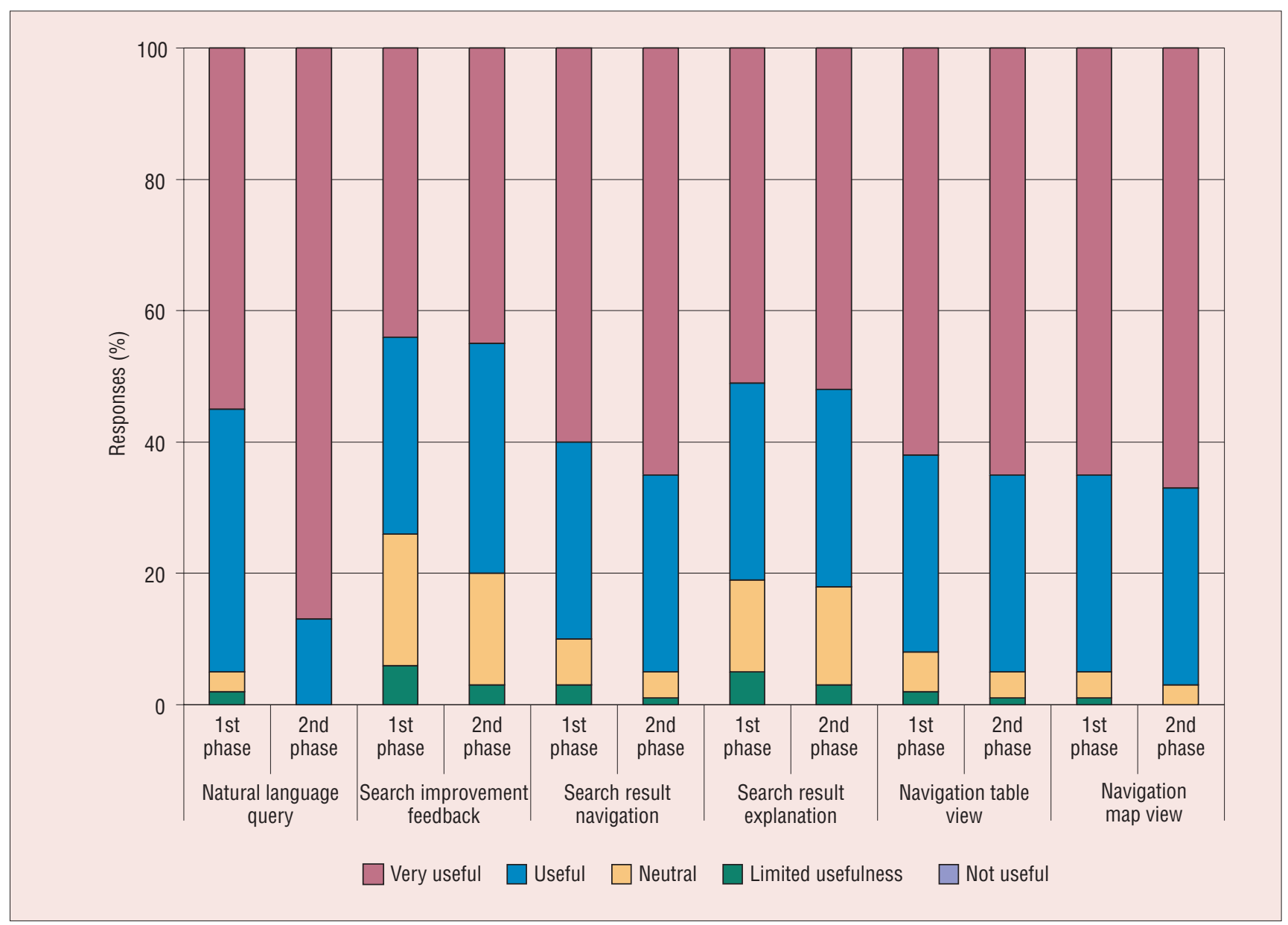

Figure 6. The increase in the perceived usefulness of INKASS over six months. Users perceived improvements to the ontology, case base, and content base primarily when using the natural language query.

ploys a mechanism for matching knowledge demands to offers that combines domain knowledge representation using a standard Semantic Web resource description and ontology language with the power and simplicity of similarity-based search employing case-based reasoning.

Third, usability-wise, it employs flexible interfaces and intelligent assistance, helping users express their demands and interactively search for appropriate offers. This assistance includes system-initiated dialogue-based retrieval, visualized topic-map navigation, clustering of retrieved results combining query-based search and naviga-

Figure 7. Evolution of the ontology, case base, and content base as a function of user transactions. User feedback captured during transactions allowed for improving the case base, the ontology, and the actual content of the knowledge exchange.

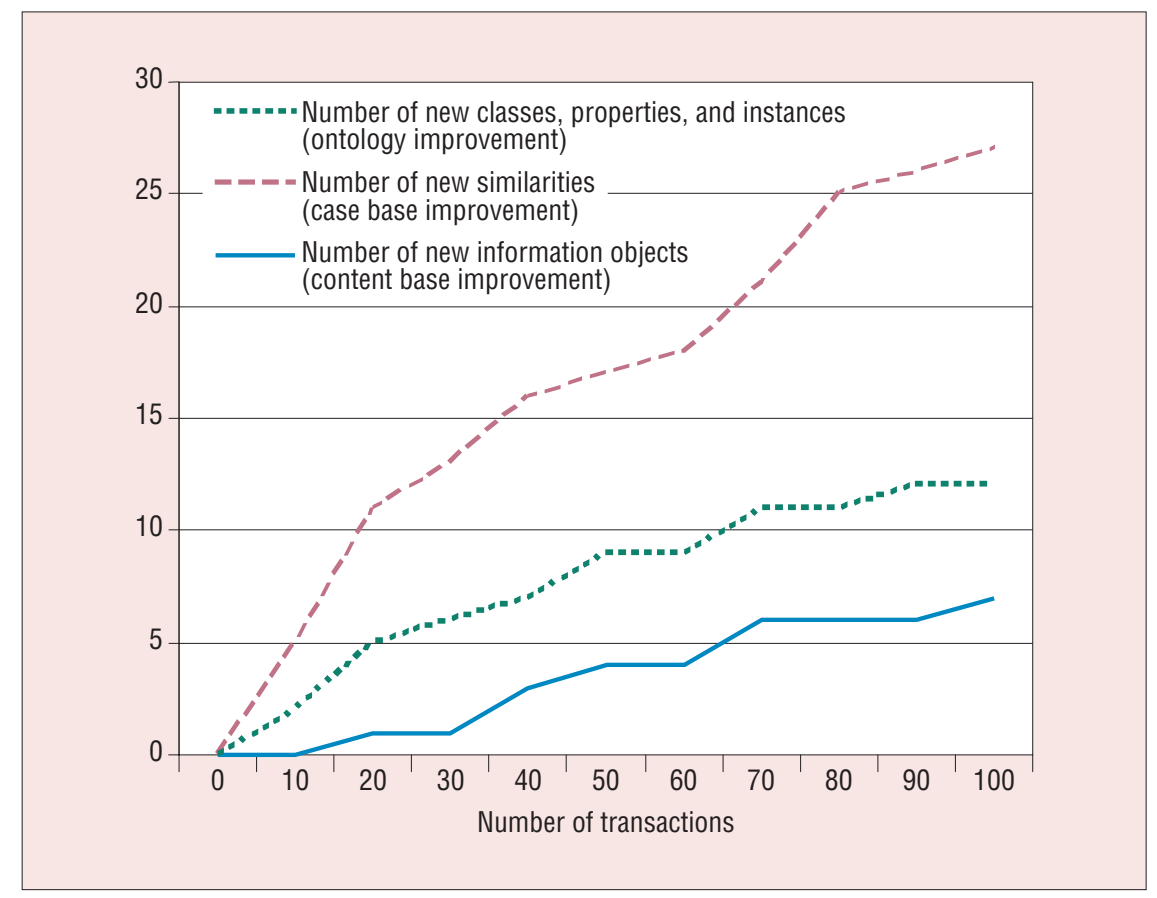




\section{The f}

Dimitris Apostolou has been the project manager of the InKASs (Intelligent Knowledge Assets Sharing and Trading) project. He's a lecturer in the Department of Informatics at the University of Piraeus and a senior researcher at the Institute of Computer and Communication Systems in Athens. His area of expertise is knowledge-based decision support systems, and his research concerns knowledge management and group decision support systems. Apostolou received his $\mathrm{PhD}$ in information systems from the National Technical University of Athens. He's a member of the IEEE. Contact him at dapost@unipi.gr.

Gregoris Mentzas is a professor in the School of Electrical and Computer Engineering at the National Technical University of Athens and the director of the Information Management Unit at the Institute of Communication and Computer Systems, Athens. His area of expertise is information technology management, and his research concerns the integration of knowledge management, the Semantic Web, and e-service technologies. Mentzas received his $\mathrm{PhD}$ in operations research and information systems from the National Technical University of Athens. Contact him at gmentzas@mail.ntua.gr.

Bertin Klein is a senior consultant at the German Research Center for Artificial Intelligence (DFKI). His research areas include knowledge representation, parsing, and pattern matching. Klein received his a Diplom in electrical engineering from Rheinisch-Westfälische Technische Hochschule Aachen. Contact him at klein@dfki.de.

Andreas Abecker is the knowledge management department manager and coordinator of the Forschungszentrum Informatik (FZI) Competence Center for Business Software. His research interests include ontology-enabled applications, the Semantic Web and Semantic Web services, and knowledge management. Abecker received his $\mathrm{PhD}$ in knowledge management systems from the University of Karlsruhe. Contact him at abecker@fzi.de.

Wolfgang Maass is a professor at the Hochschule Furtwangen University. His research interests in information management and information systems include electronic knowledge markets, paid content, dynamic pricing, and ambient intelligence, with a focus on smart products. Maass received his $\mathrm{PhD}$ from the University of Saarbrücken. He's member of the ACM. Contact him at wmaass@acm.org.

tion, and explanation of search results.

Finally, on the operational side, it leverages user transactions to improve the knowledge exchange.

Although the trial participants reported a positive experience, determining the concrete pros and cons of KESs and how per- ceived usefulness changes with respect to various factors will require a much longer operational running phase and deep empirical investigations. An open issue is whether ontologies are affordable enough to support new business paradigms, such as the one put forward here. During the trials, we found

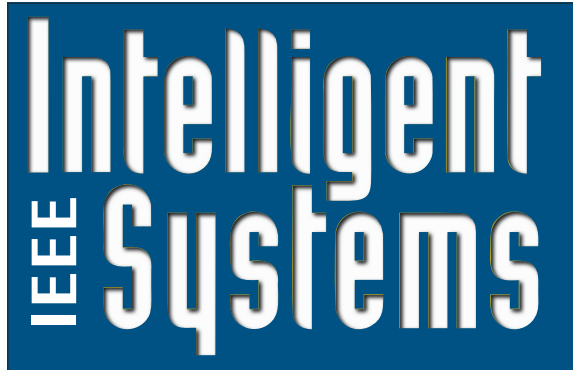

THE \#1 ARTIFIOIAL IAITELLOEEHGE MAQGAZIIIE
IEEE Intelligent Systems delivers the latest peer-reviewed research on all aspects of artificial intelligence, focusing on practical, fielded applications. Contributors include leading experts in

- Intelligent Agents • The Semantic Web - Natural Language Processing - Robotics $\bullet$ Machine Learning

Visit us on the Web at wwW.eomputer.org/intelligent that maintaining the knowledge base using the ontology-based tools (adding or deleting annotations, adding similarities, and so on) took less effort than maintaining a knowledge base of the same expressiveness in the database system that one group of participants normally used. This reduced effort might balance the initial cost of setting up the ontology. Longer-term measurements of associated costs can give a definitive answer to this issue.

Our insights have implications for practitioners as they extend their knowledge management agenda to address sharing or trading company knowledge with customers or suppliers. Our generic ontology-based approach provides a basis for modeling knowledge as an exchangeable product in an interorganizational setting. Moreover, our improvement tools can be adopted in portals that contain rich information and can help their administrators improve the portal content on the basis of users' interactions.

\section{Acknowledgments}

We acknowledge the European Commission for partially funding research presented in this article under contract IST-2001-33373. We thank all project partners, especially Ralph Traphöner of empolis, for their critical discussions and insightful contributions.

\section{References}

1. Worldwide Knowledge Management Software 2004-2008 Forecast: Access Is the Key to Growth, IDC, 2004.

2. K. Kafentzis, D. Apostolou, and G. Mentzas, "Knowledge Marketplaces: Strategic Issues and Business Models," J. Knowledge Management, vol. 8, no. 1, 2004, pp. 130-146.

3. B. Schmid and M.A. Lindemann, "Elements of a Reference Model for Electronic Markets," Proc. 31st Hawaii Int'l Conf. Systems Science (HICCs 98), IEEE CS Press, 1998, pp. 193-201.

4. G. Salton and M.J. McGill, Introduction to Modern Information Retrieval, McGrawHill, 1983.

5. B. Klein and R. Traphöner, "A Practical Application of Ontologies for Knowledge Sharing and Trading," Proc. 2004 Lernen-Wissensentdeckung-Adaptivität (LWA 2004), Humbold-Universität Berlin, pp. 259-266.

For more information on this or any other computing topic, please visit our Digital Library at www.computer.org/csdl. 